\title{
Accidente vascular encefálico en recién nacidos de término y pretérmino tardíos
}

\author{
JOSÉ LUIS MARTÍNEZ M. ${ }^{1}$, RODRIGO DÍAZ H. ${ }^{1}$, EDUARDO SÁNCHEZ C. ${ }^{2}$ \\ 1. Unidad de Neonatología. Clínica Las Condes. \\ 2. Pos-becado Pediatría. Programa de Especialización en Pediatría. Universidad de Valparaíso.
}

\begin{abstract}
\section{Brain strokes in late preterm and term newborns}

Background: Brain strokes are uncommon in term and late preterm newborns. Nevertheless, they can appear and may be diagnosed when suspected. Appropriate diagnostic techniques, available nowadays, allow a better etiologic and therapeutic approach. Objective: To report late preterm and term newborns who presented an hemorrhagic or ischemic brain stroke. Methods: Retrospective analysis of clinical charts at the Neonatology Service of Clínica Las Condes, Santiago-Chile, between January 2001 and March 2008. Results: $0.07 \%(8 / 10639)$ of these newborns presented brain stroke; 6 hemorrhagic and 2 ischemic strokes. 2 cases were diagnosed as congenital thrombophylia. No deaths were found in this survey. Conclusions: No differences in frequency were found in relation to data reported. Seizures can be the first clinical manifestation; however, subtle forms must lead to diagnostic suspicion. Appropriate diagnostic techniques may allow an accurate diagnosis and integral therapeutic approach of these patients.

(Key words: term newborn, late preterm newborn, brain stroke).

Rev Chil Pediatr 2009; 80 (1): 54-59
\end{abstract}

\section{RESUMEN}

Introducción: Los Accidentes Vasculares Encefálicos (AVE) no constituyen un diagnóstico frecuente en el grupo de Recién Nacidos de término (RNT) y pretérmino tardíos (PTT). A pesar de esto, tampoco es una situación inusual y su diagnóstico dependerá en forma importante del grado de sospecha. El uso de las apropiadas técnicas diagnósticas ha permitido una mejor caracterización de estos eventos, lo que permite en ocasiones llegar a un diagnóstico etiológico con la consiguiente optimización en el manejo. Objetivo: Caracterizar el AVE en recién nacidos de término y pretérmino tardíos. Método: Análisis retrospectivo de la ficha clínica de los pacientes RNT y RNPTT con diagnóstico de AVE nacidos entre Enero del año 2001 a Marzo del año 2008 en el Servicio de Neonatología de la Clínica Las Condes, Santiago. Resultados: Se incluyeron 8 casos, lo que corresponde al 0,07\% de la muestra estudiada ( $\mathrm{n}=10$ 639), 6 de ellos presentaron AVE hemorrágico y los 2 restantes se trataron de AVE isquémicos. En 2 casos de la serie se pesquisó

Trabajo recibido el 29 de agosto de 2008, devuelto para corregir el 14 de octubre de 2008, segunda versión el 28 de octubre de 2008, aceptado para publicación el 17 de noviembre de 2008.

Correspondencia a:

Rodrigo Díaz H.

E-mail: rodiazh@gmail.com 
trombofilia congénita. No hubo mortalidad asociada en los casos analizados. Conclusiones: La frecuencia fue similar a la de diferentes series anteriormente publicadas. La crisis convulsiva puede ser la primera manifestación clínica, pero hay otras manifestaciones clínicas más sutiles que pueden hacer sospechar el diagnóstico. El uso apropiado de las actuales técnicas diagnósticas puede llevar a un diagnóstico de certeza facilitando el manejo integral del paciente.

(Palabras clave: recién nacido de término, pretérmino tardío, accidente vascular encefálico).

Rev Chil Pediatr 2009; 80 (1): 54-59

\section{Introducción}

Los accidentes vasculares encefálicos en Neonatología han sido clásicamente referidos en la literatura a eventos hemorrágicos relacionados a prematurez o a partos traumáticos. De igual manera pueden estar asociados a asfixia perinatal, infección, trastornos de coagulación y malformaciones vasculares ${ }^{1,2}$. Básicamente se distinguen dos entidades distintas, la primera es el AVE hemorrágico que en forma habitual tiene una relación a la prematurez, pero que también puede ocurrir en niños de término y en pretérmino tardíos ${ }^{3}$, y el AVE isquémico, los que en algunas series han alcanzado una incidencia de 1 en 2300 en $\mathrm{RNT}^{4}$ y que han sido escasamente caracterizados en los reportes de la literatura nacional.

A diferencia del grupo que incluye esta serie clínica, en el prematuro se ha dado especial énfasis a la presencia de hemorragia intraventricular y sus complicaciones agudas y tardías, y su clasificación ecográfica es ampliamente conocida y relacionada al pronóstico de acuerdo al grado de hemorragia detectada ${ }^{5-7}$.

El trauma obstétrico es otra de las causas de lesiones vasculares del neonato, lo que asociado a la presencia de encefalopatía hipóxico isquémica condicionan el pronóstico futuro. Las lesiones traumáticas pueden ocasionar hemorragia subdural, subaracnoidea, intraventricular y parenquimatosa ${ }^{1,8}$.

Este tipo de lesiones ocurre con una frecuencia de 5 a 6 por cada diez mil nacidos vivos y se asocia a factores como macrosomía fetal y uso de instrumental como fórceps o vaccum extractor ${ }^{1,8,9}$. Algunas de estas condiciones requieren tratamiento quirúrgico sin embargo, la mayor parte de los casos recibe tratamiento médico conservador y seguimiento estricto por potenciales secuelas asociadas ${ }^{1,10}$.

El grupo de recién nacidos de término y de pretérmino tardío, referidos como los nacidos entre las 34 a las 36 semanas de gestación, tienen menor riesgo de presentar lesiones vasculares que los prematuros o que los nacidos por parto traumático o instrumental. Este grupo heterogéneo de pacientes ha sido escasamente estudiado y presenta características especiales que merecen ser analizadas en forma separada ${ }^{3}$. En estos pacientes la sintomatología asociada a un accidente vascular muchas veces es sutil e inespecífica por lo tanto su correcto diagnóstico requiere de un importante grado de sospecha clínica y de que se apliquen técnicas diagnósticas específicas para determinar su etiología.

El objetivo de este trabajo es presentar una serie clínica de pacientes de término y pretérmino tardíos, caracterizando su forma de presentación y estudio clínico con aproximación al diagnóstico etiológico en cada caso.

\section{Pacientes y Método}

Análisis retrospectivo de las fichas clínicas de los pacientes nacidos en Clínica Las Condes en el período comprendido entre enero del año 2001 a marzo del año 2008. El criterio de inclusión común fue el diagnóstico de Hemorragia o Infarto Cerebral en pacientes con una edad gestacional igual o mayor a 34 semanas. Los criterios de exclusión incluyeron a los pacientes nacidos en otros centros y trasladados a nuestro Servicio, los pacientes portadores de malformaciones mayores y genopatías y los pacientes prematuros con edad gestacional menor a 34 semanas. 
Cada caso fue analizado en relación a la edad gestacional, antecedentes maternos, patología del embarazo, tipo de parto e instrumentalización del parto. Igualmente se consignaron los antecedentes de cada recién nacido en relación a peso de nacimiento, test de Apgar, vía del parto y la instrumentalización en el parto. Se registraron las manifestaciones clínicas, y los estudios de laboratorio realizados con énfasis en neuroimágenes y en el estudio de trombofilia que incluyó: Proteína C, Antitrombina III, resistencia a la Proteína $\mathrm{C}$ activada, Anticoagulante lúpico, Proteína S libre, Homocisteína y desde el año 2005 PCR para Factor V de Leiden y mutación del gen de Protrombina. Los resultados del análisis de los casos se presentan en forma descriptiva.

\section{Resultados}

Durante el período estudiado nacieron 11060 recién nacidos vivos. Un total de 65 pacientes no fueron incluidos en esta revisión por presentar malformaciones y genopatías incompatibles con la vida. Un total de 356 pacientes prematuros con edad gestacional menor a 34 semanas fueron excluidos del estudio, lo que deja un total de 10639 pacientes incluidos en el análisis final, seleccionándose 8 casos con diagnóstico de AVE hemorrágico o isquémico, lo que equivale a $0,07 \%$ de la muestra que resulta en 1 afectado de cada 1329 niños. Los pacientes que cumplieron con los criterios de inclusión fueron ocho en total, desglosándose en dos casos de AVE isquémico y seis de AVE hemorrágico.

Los pacientes con AVE isquémico se detallan en la tabla 1 destacando que ambos casos se presentaron como convulsiones neonatales dentro de las primeras 48 horas de vida y cuyo estudio en ambos correspondió a infartos de territorio silviano. El estudio de trombofilia mostró una mutación del factor $\mathrm{V}$ de Leiden en uno de los casos. La presencia de AVE isquémico afectó a 1 de cada 5319 niños.

Las características de los 6 pacientes con AVE hemorrágico se detallan en la tabla 2 destacando que la forma de presentación inicial fue polimorfa y no siempre a través de cuadros convulsivos, encontrándose como primera manifestación clínica alteraciones como crisis de cianosis o trastorno de deglución. En uno de los casos se pesquisó dilatación ventricular prenatal a las 34 semanas de edad gestacional. Las hemorragias se localizaron de preferencia a

Tabla 1. Características de los pacientes con AVE isquémico

\begin{tabular}{lll}
\hline Pacientes & $\mathbf{1}$ & $\mathbf{2}$ \\
\hline Edad gestacional & 38 sem & 39 sem \\
Vía del parto & Vaginal & Vaginal \\
Uso de fórceps & No & No \\
Apgar 1 y 5 min & $9 / 10$ & $8 / 9$ \\
Gases de cordón & No realizados & No realizados \\
Primera manifestación clínica & Convulsión & Convulsión \\
Edad al presentar primera manifestación clínica & 48 horas de vida & 30 horas de vida \\
Territorio & Infarto silviano posterior derecho & Infarto silviano izquierdo \\
Ecografía encefálica & Normal & Normal \\
TAC & No realizado & No realizado \\
RM & Infarto silviano & Infarto silviano \\
EEG & Foco epileptiforme centro temporal & Actividad epileptiforme \\
Estudio trombofilia & Negativo & Mutación factor V de Leiden \\
Estudio metabólico & Normal & Normal \\
Ecocardiografía & Normal & Normal \\
Secuelas & Epilepsia 2 & Epilepsia $2^{\circ}$ \\
\hline
\end{tabular}


Tabla 2. Características AVE (hemorragia)

\begin{tabular}{|c|c|c|c|c|c|c|}
\hline Pacientes & 1 & 2 & 3 & 4 & 5 & 6 \\
\hline Edad gestacional & 34 sem & 37 sem & 36 sem & 36 sem & 40 sem & 38 sem \\
\hline $\begin{array}{l}\text { Diagnóstico } \\
\text { prenatal }\end{array}$ & No & No & No & Sí & No & No \\
\hline Vía del parto & Vaginal & Vaginal & Vaginal & Cesárea & Vaginal & Vaginal \\
\hline Uso de fórceps & No & No & No & No & No & No \\
\hline Apgar & $9 / 9$ & $9 / 10$ & $8 / 9$ & $9 / 10$ & $9 / 9$ & $8 / 9$ \\
\hline Gases cordón & No & No & No & No & No & No \\
\hline $\begin{array}{l}\text { Primera mani- } \\
\text { festación clínica }\end{array}$ & Convulsión & Convulsión & Crisis de cianosis & $\begin{array}{l}\text { Trastorno } \\
\text { deglución }\end{array}$ & Crisis de cianosis & $\begin{array}{l}\text { Hipotonia } \\
\text { Crisis de cianosis }\end{array}$ \\
\hline $\begin{array}{l}\text { Edad al presentar } \\
\text { primera manifes- } \\
\text { tación clínica }\end{array}$ & $2^{\circ}$ día & $4^{\circ}$ día & $5^{\circ}$ día & $1^{\circ}$ día & $1^{\circ}$ día & $7^{\circ}$ día \\
\hline $\begin{array}{l}\text { Ecografía } \\
\text { encefálica }\end{array}$ & $\begin{array}{l}\text { HIVD Go IV y } \\
\text { HIVI Go III }\end{array}$ & HIVD Go III & $\begin{array}{l}\text { HIVD Go I } \\
\text { HIVI G० । }\end{array}$ & HIV Go IV & $\begin{array}{l}\text { Hemorragia } \\
\text { leptomeningea }\end{array}$ & HIV Go III \\
\hline TAC & $\begin{array}{l}\text { Hidrocefalia } \\
\text { cuadriventricular }\end{array}$ & Hidrocefalia & No realizado & No realizado & $\begin{array}{l}\text { Hemorragia } \\
\text { leptomeningea }\end{array}$ & No realizado \\
\hline RM & No realizado & $\begin{array}{l}\text { Hemorragia } \\
\text { intraventricular }\end{array}$ & No realizado & $\begin{array}{l}\text { Hemorragia } \\
\text { intraventricular }\end{array}$ & $\begin{array}{l}\text { Hemorragia } \\
\text { leptomeningea }\end{array}$ & No realizado \\
\hline EEG & $\begin{array}{l}\text { Actividad epilep- } \\
\text { tiforme frontal } \\
\text { derecha }\end{array}$ & $\begin{array}{l}\text { Actividad epilep- } \\
\text { tiforme frontal } \\
\text { derecha }\end{array}$ & Normal & Normal & $\begin{array}{l}\text { Actividad } \\
\text { paroxística } \\
\text { centro-temporal }\end{array}$ & No realizado \\
\hline $\begin{array}{l}\text { Estudio } \\
\text { trombofilia }\end{array}$ & $\begin{array}{l}\text { Homocigocidad } \\
\text { del polimorfismo } \\
\text { A } 1298 \text { C de la } \\
\text { MTHFR(*) }\end{array}$ & Negativo & No realizado & Negativo & Negativo & No realizado \\
\hline $\begin{array}{l}\text { Estudio } \\
\text { Metabólico }\end{array}$ & Normal & Normal & Normal & Normal & Normal & Normal \\
\hline Ecocardiografía & Normal & Normal & Normal & Normal & $\begin{array}{l}\text { Hipertensión } \\
\text { pulmonar } \\
\text { transitoria }\end{array}$ & No realizado \\
\hline Secuelas & $\begin{array}{l}\text { Hidrocefalia } \\
\text { Epilepsia }\end{array}$ & $\begin{array}{l}\text { Hidrocefalia } \\
\text { Epilepsia }\end{array}$ & No & $\begin{array}{l}\text { Trastorno } \\
\text { deglución }\end{array}$ & No & No \\
\hline
\end{tabular}

HIVD: Hemorragia intraventricular derecha; HIVI: Hemorragia intraventricular izquierda; *Metilentetrahidrofolato reductasa.

nivel intraventricular. En uno de los casos se pesquisó una alteración de la metilentetrahidrofolato reductasa. La presencia de AVE hemorrágico afectó a 1 de cada 1773 niños.

\section{Discusión}

La frecuencia mostrada en esta serie es de 1 cada 1329 recién nacidos lo cual es similar en relación a lo informado en diferentes publicaciones, siendo el AVE hemorrágico tres veces más frecuentemente observado ${ }^{11}$.
Con respecto a los AVE isquémicos es concordante con la literatura la manifestación clínica cardinal que es la convulsión. Además, son similares a las series descritas el territorio comprometido y la unilateralidad de los eventos ${ }^{11,12}$.

Dentro del estudio destacan tres pilares fundamentales: la Resonancia Magnética (RM), el uso de Video EEG, y el estudio de trombofilia.

$\mathrm{Al}$ igual que series anteriormente publicadas la utilidad de la Ecografía Encefálica en AVE isquémicos fue nula, a pesar de ser realizada con equipos adecuados y con médicos radiólogos especialistas, por lo cual parece indispensable 
realizar una RM si el índice de sospecha es alto y la Ecografía encefálica no arroja resultados esclarecedores ${ }^{4,13}$.

El uso del Video EEG impresiona en estos casos con una utilidad múltiple, ya que no sólo puede confirmar la actividad epileptiforme sino que también puede dar una orientación del territorio comprometido, en especial en caso de no contar con la disponibilidad inmediata de una neuroimagen ${ }^{14,15}$. Además, será útil en la monitorización del cuadro convulsivo y subsecuente ajuste de medicamentos anticonvulsivantes ${ }^{16}$.

Como tercer pilar destaca el estudio de trombofilia que puede llevar a diagnóstico etiológico con la consiguiente optimización del manejo y mayor claridad sobre el pronósti$\mathrm{co}^{17,18}$. Estas evaluaciones pueden ser complementadas con estudio ecocardiográfico y de enfermedades metabólicas.

En relación al grupo con AVE hemorrágicos llaman la atención algunas de sus características. Si bien es cierto que las manifestaciones clínicas dependerán de la localización de la hemorragia, la gravedad y la edad gestacional del paciente hay signos que destacan como clásicos: convulsiones, irritabilidad, depresión de nivel de conciencia y déficits neurológicos focales. En esta serie sólo dos de los pacientes presentaron crisis convulsivas, el resto tuvo manifestaciones más bien sutiles como crisis de cianosis o alteración de deglución.

En esta serie no se encontró asociación con factores de riesgo prenatales como infección, tampoco con factores intraparto como trauma obstétrico o asfixia perinatal, ni con factores neonatales como trastornos respiratorios o hemodinámicos.

Con respecto al tipo de hemorragia se pesquisó con mayor frecuencia hemorragia intraventricular de reconocida mayor incidencia en los menores de 32 semanas en desmedro de la hemorragia subdural y epidural habitualmente relacionadas a $\mathrm{RNT}^{19}$. El momento de presentación está dentro de los rangos descritos como habituales y generalmente corresponde a las primeras 48 horas de vida.

Con respecto al método diagnóstico la ecografía encefálica confirma su valor en el diagnóstico y en el posterior seguimiento de los casos de AVE hemorrágicos de la serie.
El estudio de trombofilia nuevamente resultó fundamental en al menos uno de los casos (al igual que AVE isquémicos) y permitió tratamiento y seguimiento específico.

En síntesis, podemos decir que los AVE si bien son poco frecuentes, no son eventos aislados en RNT y RNPTT. Su adecuada pesquisa dependerá de una alta sospecha clínica ya que aunque hay factores de riesgo conocidos y manifestaciones clínicas clásicas, la forma de presentación clínica puede ser mucho más sutil de lo descrito en forma habitual. En el caso de sospecha de AVE isquémico impresiona fundamental contar con una neuroimagen de alta calidad ya que el rendimiento de la Ecografía es pobre, no así en AVE hemorrágico donde la Ecografía encefálica es una excelente herramienta de diagnóstico, seguimiento e incluso de orientación etiológica ${ }^{20}$.

El uso de Video EEG parece clave en la confirmación y seguimiento de la actividad convulsiva. Por último, el estudio de trombofilia resulta un complemento necesario, ya que puede llevar a diagnóstico etiológico, lo que contribuye a un manejo adecuado.

El seguimiento clínico especializado y multidisciplinario de estos pacientes es fundamental para optimizar su desarrollo integral y neurológico e igualmente importante es el apoyo a los padres tanto en la fase aguda como frente a las potenciales secuelas futuras de estas patologías.

\section{Conclusión}

La frecuencia de AVE en esta serie coincide a otras experiencias publicadas. Aunque la crisis convulsiva puede ser la primera manifestación clínica, existen otros elementos clínicos más sutiles que deben ser considerados. El correcto uso de las actuales técnicas diagnósticas puede llevar a un diagnóstico de certeza y facilitar el manejo integral del paciente.

\section{Referencias}

1.- Doumouchtsis S, Arulkumaran S: Head trauma after instrumental births. Clin Perinatol 2008; 35: 69-83.

2.- Pollina J, Días MS, Li V: Cranial births injuries in 
term newborn infants. Pediatr Neurosurg 2001; 35: 113-9.

3.- Engle W, Tomashek K, Wallman C: Neonatos pretérmino tardíos : una población en riesgo. Pediatrics (Ed esp) 2007; 64 (6): 357-66.

4.- Schulzke S, Weber P, Luetschg J, Fahnenstich H: Incidence and diagnosis of unilateral arterial cerebral infarction in newborn infants. J Perinat Med 2005; 33: $170-5$.

5.- Roland E, Hill A: Intraventricular hemorrhage and posthemorrhagic hydrocephalus. Clin Perinatol 1997; 24: 589-605.

6.- Brouwer A, Groenendaal F, van Haastert IL, Rademaker K, Hanlo P, de Vries L: Neurodevelopmental outcome of preterm infants with severe intraventricular hemorrhage and therapy for posthemorrhagic ventricular dilatation. J Pediatr 2008; 152: 648-54.

7.- Volpe JJ: Brain injury in the preterm infant : Overview of clinical aspects, neuropathology and pathogenesis. Semin Pediatr Neurol 1998; 5: 135-51.

8.- Jhawar BS, Ranger A, Steven D: Risk factors for intracranial hemorrhage among full-term infants: a case-control study. Neurosurgery 2003; 52: 581-90.

9.- Towner D, Castro MA, Eby-Wilkens E: Effect of mode of delivery in nulliparous women on neonatal intracranial injury. N Engl J Med 1999;341:1709-14

10.- Negishi H, Lee Y, Itoh K: Nonsurgical management of epidural hematoma in neonates. Pediatr Neurol 1989; 5: 253-6.

11.- De Vries L: Intracraneal Hemorrhage and Vascular Lesions. Fanaroff and Martin's. Neonatal-Perinatal Medicine. 8th edition. Philadelphia. Mosby 2006: 934-7.
12.- Devilat BM, Lamas GR, Castro AM: Infarto cerebral en recién nacidos a termino con convulsiones. Rev Chil Pediatr 1988; 59: 334-8.

13.- Golomb MR, de Veber G, McGregor DL, Domit et al: Cranial ultrasonography has a low sensitivity for detecting arterial ischemic stroke in term neonates. $\mathrm{J}$ Child Neurol 2003: 18:98.

14.- Koelfen $W$, Freund $M$, Varnholt $V$ : Neonatal stroke involving the middle cerebral artery in term infants: clinical presentation, EEG and imaging studies, and outcome. Dev Med Child Neurol 1995; 37: 204-12.

15.- Marret S, Lardennois C, Mercier A, et al: Fetal and Neonatal Cerebral Infarcts. Biol Neonate 2001; 79: 236-40.

16.- Boylan G, Rennie J, Pressler R, et al: Phenobarbitone, neonatal seizures, and video-EEG. Arch Dis Child Fetal Neonatal Ed. 2002; 86 (3): F165-70.

17.- Günther $G$, Junker $R$, Sträter $R$, et al: Symptomatic Ischemic Stroke in Full-Term Neonates: Role of Acquired and Genetic Prothrombotic Risk Factors. Stroke 2000; 31: 2437-41.

18.- Mercuri E, Cowan F, Gupte G, et al: Prothrombotic Disorders and Abnormal Neurodevelopmental Outcome in Infants With Neonatal Cerebral Infarction. Pediatrics 2001; 107: 1400-4.

19.- Soul J: Hemorragia intracraneal. Cloherty Manual de Cuidados Neonatales. $4^{\text {a }}$ edición. Barcelona. Masson, 2005: 604-619.

20.- Leijser LM, de Vries LS, Rutherford MA, et al: Cranial Ultrasound in Metabolic Disorders Presenting in the Neonatal Period: Characteristic Features and Comparison with MR Imaging. AJNR Am J Neuroradiol 2007; 28: 1223-31. 